10 years ESJ

Special edition

\title{
Servicio de Entrega: Tendencia de Hábitos y Consumo de Comida Mediante el uso de Aplicaciones Móviles
}

\author{
Fátima Lizette Reyes Perzabal \\ Tecnológico Nacional de México Campus Tehuacán, Puebla, México \\ Jesús Alberto Gómez Peralta \\ Rogelio Vázquez Quitl \\ Helen Javier Chávez Guzmán \\ Universidad Popular Autónoma del Estado de Puebla, Mexico
}

Doi:10.19044/esj.2021.v17n4p96

Submitted: 28 October 2020

Accepted: 29 November 2020

Published: 07 February 2021
Copyright 2021 Author(s)

Under Creative Commons BY-NC-ND

4.0 OPEN ACCESS

Cite As:

Reyes Perzabal F.L., Gómez Peralta J.A., Vázquez Quitl R. \& Chávez Guzmán H.J. (2021).

Servicio de Entrega: Tendencia de Hábitos y Consumo de Comida Mediante el uso de Aplicaciones Móviles. European Scientific Journal, ESJ, 17(4), 96.

https://doi.org/10.19044/esj.2021.v17n4p96

\section{Resumen}

El uso de las aplicaciones móviles para pedir comida se ha tornado en algo natural para el consumidor que vive en la contingencia causada por COVID-19, sin embargo, no se ha convertido en un hábito ni ha generado dependencia o adicción alguna para tal fin. El enfoque de la presente investigación es cuantitativo de alcance descriptivo, transversal simple. Como técnica de recolección se empleó la encuesta electrónica por internet, aplicándose a 75 personas habitantes de la ciudad de Puebla que utilicen aplicaciones móviles de entrega de alimentos, la cual se conforma por 32 ítems. Se analizó el perfil del consumidor de comida a través de aplicaciones móviles, sus hábitos y actitudes hacia el servicio, con la finalidad de generar estrategias de comercialización a través de la segmentación. Se detectó que factores como el ahorro de tiempo, dinero y rapidez en la entrega de alimentos son valorados por la mayoría; no obstante, existe claramente un segmento de mercado que posee características que lo distinguen en cuanto al perfil y gasto se refiere. El uso promedio es de una sola aplicación, siendo Uber Eats la que más demanda tiene, el momento de mayor frecuencia para la compra de alimentos es por la noche, con un gasto menor a los $\$ 500$ al mes y la razón principal, es para no 
salir de casa.

Palabras clave: Comportamiento Del Consumidor, Segmentación De Mercados, Aplicaciones Móviles Para Pedir Comida, COVID-19

\title{
Delivery Service: Trend in Habits and Food Consumption Through the use of Mobile Applications
}

\author{
Fátima Lizette Reyes Perzabal \\ Tecnológico Nacional de México Campus Tehuacán, Puebla, México \\ Jesús Alberto Gómez Peralta \\ Rogelio Vázquez Quitl \\ Helen Javier Chávez Guzmán \\ Universidad Popular Autónoma del Estado de Puebla, Mexico
}

\begin{abstract}
The use of mobile applications to order food has become something natural for the consumer who lives in the contingency caused by COVID-19. It, however, has not developed into a habit, neither has it generated any dependency nor addiction for this purpose. This paper uses a quantitative approach, descriptive in scope, and follows a simple transversal. As a collection technique, the electronic survey was used on the internet and it was applied to 75 inhabitants of the city of Puebla that use mobile applications for food delivery, which is made up of 27 items. The profile of the food consumer, their habits and attitudes towards the service, was analyzed through mobile applications in order to generate marketing strategies through segmentation. The results show that factors such as saving time, money, and the speed of food delivery are valued by most people. There is also a clear market segment that has features that distinguish it in terms of profile and spending. The average use is just a single app, i.e., Uber Eats - the one with the most demand. People buy food more frequently at night, with an expense of less than $\$ 500$ a month due to home delivery.
\end{abstract}

Keyword: Consumer Behavior, Market Segmentation, Mobile Food Ordering Apps, COVID-19

\section{Introducción}

El 2020 será un año que siempre se recordará debido a la situación de la pandemia mundial generada por el virus COVID- 19 que ha impactado todas las áreas posibles, desde la personal, social, familiar, educativa, económica hasta incluso la forma en la que las personas consumen. Los consumidores 
ahora viven, compran y piensan de manera diferente. Los hábitos implicarán soportar más allá de esta crisis, cambiando permanentemente lo que se valora, cómo y dónde se compra y cómo se vive y trabaja (Verma \& Gustafsson, 2020). Desde el inicio, se observó que esta pandemia había acelerado fenómenos y tendencias a largo plazo, anticipó cambios que se habían contemplado en los próximos años a tan sólo semanas, obligando a las empresas a adaptarse (González Vaqué, 2020).

Un sector que se ha visto afectado en todo el mundo es el restaurantero, dado que las medidas sanitarias han prohibido la apertura de espacios sociales por ser un foco de propagación del virus. Esta situación ya ha cobrado la factura para muchos, llevándolos a la quiebra y por consiguiente el despido de todo el personal que labora en estos espacios ha sufrido las consecuencias. Según Brizek et al. (2020), el 25\% de los restaurantes encuestados en un estudio afirmaron que no sobrevivieron al cierre de casi dos meses, menos de una cuarta parte no pudieron regresar a los empleados de su empresa a los niveles previos a la pandemia y aproximadamente el $65 \%$ no creía poder mantener abierto su restaurante si las restricciones pandémicas permanecían vigentes hasta 2021.

En lo que respecta al consumo en restaurantes, en Estados Unidos el $3 \%$ de los operadores de éstos cerraron permanentemente, el $44 \%$ lo hizo temporalmente y el $11 \%$ anticipa que cerrará permanentemente, lo que representó que las ventas cayeran un 47\% durante el período del 1 al 22 de marzo (New research details early impact of coronavirus pandemic on restaurant industry, 2020). Por otra parte, el escenario en México mostró en marzo una caída drástica en el consumo en restaurantes de $280.94 \%$, en abril de $127.02 \%$ y en mayo de $125.71 \%$ (Reveles, 2020).

Sin embargo, ante esta repentina situación, el sector de restaurantes comerciales está luchando por reorganizarse. La resiliencia es crucial para que un sistema pueda responder adecuadamente a eventos de esta magnitud y se dirija a la recuperación y adaptación ante las adversidades (Gomes \& Stedefeldt, 2020). Como medidas de adaptación, muchos restaurantes han optado por migrar o tener mayor presencia en las aplicaciones móviles de entrega de comida. De acuerdo a Reveles (2020), los mexicanos redujeron drásticamente su consumo en restaurantes, en tanto que aumentaron el uso de servicios de entrega a domicilio, impulsando a Rappi y Uber Eats como las apps ganadoras durante el coronavirus. De acuerdo con Forbes México (2020), las plataformas de comercio electrónico han crecido en promedio $300 \%$ tanto en aumento de pedido como en socios restauranteros. Por lo tanto, es innegable el impacto que el COVID-19 ha tenido en los bienes de consumo provocando efectos permanentes. A medida que las empresas avanzan, deben considerar cómo comprender mejor a los consumidores y esforzarse para superar la incertidumbre en esta nueva era (Verma \& Gustafsson, 2020). 
De lo anterior, queda de manifiesto que entender el fenómeno de consumo resulta valioso en estos tiempos de crisis. Las recientes investigaciones señalan que existen cambios en la forma en la que están consumiendo las personas en el mundo, por ello, resulta crucial y oportuno estudiar el fenómeno en un espacio local, especialmente para el sector restaurantero. Así mismo, los datos recabados en esta investigación permiten generar estrategias con el fin de lograr ventajas competitivas a partir de comprender la relación entre los restaurantes y el servicio de entrega de alimentos, identificando el cambio en los hábitos del consumo de comida mediante el uso de estas aplicaciones.

\section{Objetivos}

Analizar el perfil del consumidor de comida a través de aplicaciones móviles, sus hábitos y actitudes hacia el servicio en la ciudad de Puebla, para generar estrategias de comercialización a través de la segmentación.

Los objetivos específicos, son los siguientes:

1. Determinar los factores que influyen en el pedido del consumidor de comida a través de aplicaciones móviles.

2. Conocer las preferencias de los consumidores sobre el pedido de comida a través de aplicaciones móviles.

3. Identificar los cambios en los hábitos de compra y consumo de comida de los consumidores como consecuencia del COVID-19.

La revisión de la literatura se enfocó en los siguientes conceptos: mercadotecnia, comportamiento del consumidor, segmentación de mercados, contexto del sector restaurantero y aplicaciones de entrega de comida y nuevos comportamientos del consumidor a partir de COVID-19.

Entre los aspectos más significativos de dicha revisión, destaca que en México sólo el $15 \%$ de todos los restaurantes se ha adaptado y cuenta con el servicio de entrega a domicilio o recoger directamente (pick-up) (CANIRAC, 2020). Desde el 2017 el servicio de entrega de alimentos ha crecido entre los consumidores debido a ciertos factores como la comodidad al no salir de casa, la rapidez en el servicio y la variedad de restaurantes que se ofertan por parte de las plataformas y de acuerdo a estimaciones de Euromonitor, se calcula que para 2021, las aplicaciones de entrega de comida tendrán ingresos al año por 15 mil millones de pesos. Con datos proporcionados por Uber Eats, Rappi y DiDi Food, se establece que un mexicano pide comida a través de estas aplicaciones alrededor de cuatro veces al mes, gastando aproximadamente 700 pesos (Mulato, 2019), lo cual se puede observar en la Figura 1.

Sin embargo, el gasto aumenta con la edad, ya que los menores de 25 años destinan en promedio $\$ 140$ pesos para este servicio, mientras que usuarios de entre 25 y 34 años $\$ 180$ pesos. Esta cifra se eleva a usuarios de un rango de edad que va de los 35 a 44 años con un promedio de $\$ 220$ pesos, 
mientras que los consumidores mayores a 45 años desembolsan alrededor de $\$ 340$ pesos (Reveles, 2019). En este mismo sentido, a pesar de que la comida que más solicitan los mexicanos es la cena, el desayuno ha tomado relevancia entre los usuarios y el sábado se posiciona como el día en que más gastan los mexicanos a través de este tipo de aplicaciones (Galeano, 2019).

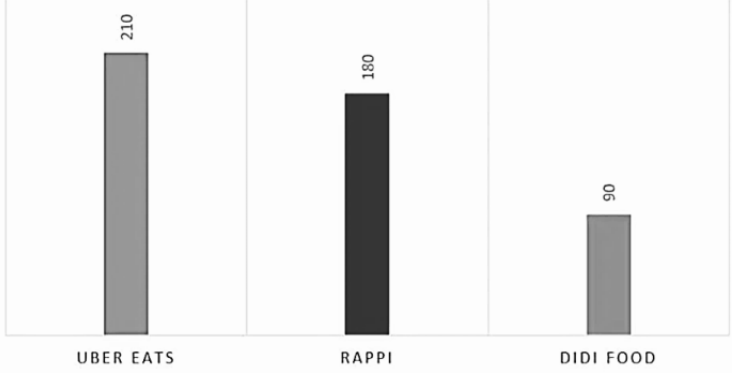

Ticket promedio gastado por un mexicano en

Figura 1. Gasto promedio en aplicaciones de entrega de comida para un mexicano. Tomado de Goula Especialistas en la Industria alimenticia (Mulato, 2019).

En lo que concierne al sector restaurantero, hasta ahora, la transmisión de SARS-CoV-2, a través de alimentos no ha sido confirmada. No obstante, esta situación no los exime de adoptar medidas de seguridad e higiene en la producción de alimentos (Gomes \& Stedefeldt, 2020). Algunas de las prácticas novedosas que han optado los operadores de todo el mundo incluyen nuevos formatos que eliminan las áreas de descanso o fomentan el consumo en otros lugares como una alternativa a los restaurantes para sentarse. Estas innovaciones vienen en respuesta a la mano de obra adicional para desinfectar las áreas de descanso, los mandatos de distanciamiento social y la renuencia de algunos a volver a espacios públicos cerrados, por lo que los consumidores están volviendo a usar el sitio web o las aplicaciones cuando hacen pedidos en línea (Winsight, 2020).

\section{Metodología}

Se desarrolló una investigación cuantitativa, no experimental dado que en esta investigación no se manipulan las variables, sino que se observan cómo se dan en el contexto natural (Hernández, Fernández y Baptista, 2014). Es de tipo transversal simple, debido a que la recolección se ha realizado una sola vez en un periodo de tiempo y con un solo tipo de muestra. Es descriptiva, ya que busca describir las características de las variables estudiadas.

La técnica de recolección de datos fue encuesta personal por internet, realizada a través de Google Forms. Los sujetos de estudio fueron habitantes de la ciudad de Puebla que utilizan aplicaciones móviles de entrega de alimentos como Uber Eats, Rappi y Sin Delantal, para pedir comida. Se utilizó la fórmula para poblaciones infinitas, considerando que para el año 2020 en la ciudad de Puebla hay 1,539,819 personas, según datos de INEGI. El nivel de 
confianza fue del $90 \%$ y el margen de error del $10 \%$, arrojando una muestra de 75 individuos, a los cuales se les envió el cuestionario entre el 2 y el 10 de julio del presente año. Con la información recopilada se elaboró una base de datos en Excel. Posteriormente, los datos fueron procesados utilizando el programa SPSS con el fin de determinar las relaciones entre las distintas variables. Los resultados obtenidos se presentan a través de tablas y figuras con sus respectivos porcentajes, así como la descripción e interpretación de los datos estadísticos, para de esta forma, cumplir con los objetivos propuestos.

Para llevar a cabo la recolección de datos, se utilizaron 32 ítems, incluida la información demográfica. Con la operacionalización de nueve variables se logró la construcción del cuestionario de acuerdo a los requerimientos de la investigación. Dichas variables son Perfil de uso de la aplicación móvil de entrega de comida (Densingh, 2019), Hábitos, Orientación para ahorrar tiempo y Actitud hacia los servicios de entrega de comida (Yeo, Goh, \& Rezaei, 2017), Frecuencia de uso y Precio/Ahorro, (Lee, Sung \& Jeon, 2019), Variedad (Azizul et al., 2019), Higiene (Kim Dang et al., 2018) y Cambio en los hábitos de Alimentación y/o Consumo de comida como resultado de COVID-19 (Food Insight, 2020).

\section{Hallazgos}

Los hallazgos se basaron en la información recopilada de la encuesta. La mayor participación fue femenina, equivaliendo al $61 \%$, el $28 \%$ se encuentra en un rango de edad entre 28 y 32 años, seguido por el $25 \%$, entre 17 y 22 años. En cuanto al nivel de ingresos familiares, el 52\% percibe entre $\$ 4,501$ y $\$ 10.600$, el $25 \%$ entre $\$ 10,601$ y $\$ 17,000$, que representan los mayores porcentajes. Con relación al nivel de estudios, el $64 \%$ cuenta con maestría y el $22 \%$ con licenciatura.

Tabla 1. Perfil demográfico de la muestra

\begin{tabular}{llcc} 
& & N & \% \\
\hline \multirow{2}{*}{ Sexo } & Hombre & 29 & $38.7 \%$ \\
& Mujer & 46 & $\mathbf{6 1 . 3} \%$ \\
Edad & $17-22$ & & \\
& $23-27$ & 19 & $25.3 \%$ \\
& $28-32$ & 16 & $21.3 \%$ \\
& $33-39$ & 21 & $\mathbf{2 8 . 0} \%$ \\
Nivel de Estudio & $42-49$ & 11 & $14.7 \%$ \\
& $53-55$ & 5 & $6.7 \%$ \\
& Preparatoria & 3 & $4.0 \%$ \\
& Licestria & 8 & $10.7 \%$ \\
& Doctorado & 48 & $\mathbf{6 4 . 0} \%$ \\
Ingreso mensual & Menos de $\$ 4.500$ & 2 & $2.7 \%$ \\
familiar & Entre $\$ 4,501$ y $\$ 10,600$ & 39 & $\mathbf{5 2 . 0} \%$ \\
& Entre $\$ 10,601$ y $\$ 17,000$ & 19 & $\mathbf{2 5 . 3 \%}$ \\
& Entre $\$ 30,000$ y $\$ 29,000$ & 2 & $2.7 \%$ \\
& Entre $\$ 81,000$ y $\$ 250,000$ & 4 & $5.3 \%$ \\
& Más de 250.000 & 1 & $1.3 \%$ \\
& & 1 & $1.3 \%$
\end{tabular}

Nota: Tomado de Goula Especialistas en la Industria alimenticia (Goula, 2019) 
El análisis de las variables antes mencionadas permitió conocer el perfil del usuario de aplicaciones móviles para la entrega de comida, en donde se establece que el $48 \%$ de las personas sólo utilizan una aplicación, siendo Uber Eats, con el 52\%, la app de mayor preferencia. La comida del día en la que se utiliza con mayor frecuencia una aplicación móvil para solicitar alimentos es la cena (52\%). El 62.7\% gasta en promedio al mes menos de $\$ 500$ para pedir alimentos por este medio y la razón principal es evitar salir de casa, con el 36\%. Quien toma la decisión de ordenar comida de esta manera es el propio encuestado (yo, consumo personal) reflejado en el $53.3 \%$ de los encuestados.

Respecto a la variable Hábito, comprar comida a través de aplicaciones de entrega de alimentos no se ha convertido meramente en un hábito, ya que los encuestados se muestran indiferentes en adquirirlos por medio de la aplicación o salir a comprarlos, lo cual está sustentado por el 37.3\%. Así mismo, el 56\% refleja total desacuerdo al considerarse adicto o dependiente del uso de aplicaciones para adquirir sus alimentos. El $41.3 \%$ está totalmente en desacuerdo en considerar que debe usar aplicaciones de entrega de alimentos para adquirir su comida, sin embargo, para el $25.3 \%$ el usar estas aplicaciones para tal fin se ha vuelto algo natural.

Para la variable Orientación para ahorrar tiempo, el 36\% se encuentra en total desacuerdo al pensar que el uso de los servicios de aplicación móvil de entrega de comida es muy útil en el proceso de compra, por el contrario, el $32 \%$ está totalmente de acuerdo en que les ayuda a lograr más rápidamente el proceso de compra.

Así mismo, el $41.3 \%$ sostiene estar totalmente de acuerdo en que puede ahorrar tiempo y para el $33.3 \%$ es importante que la compra de alimentos se realice lo más rápido posible utilizando dichos servicios.

La variable de Actitud, que tiene por objeto conocer en qué grado se ubica la percepción del consumidor respecto a si es prudente, bueno, razonable y gratificante el comprar alimentos a través de los servicios de aplicación móvil de entrega de comida determina que el $34.7 \%$ es indiferente a la hora de preguntarse si es prudente hacer este tipo de compra, el 36\% está de acuerdo con que es una buena idea pedir comida por este tipo de aplicaciones, del mismo modo el $36 \%$ se encuentra indiferente ante si piensa que es razonable adquirir los productos por este medio y existe una fuerte similitud con el $32 \%$ de quienes son indiferente y están de acuerdo en encontrar gratificante adquirir alimentos por medio de este servicio de comida a domicilio.

La variable Frecuencia de uso de la aplicación móvil, permitió conocer que la mayor parte de los encuestados, el 53.3\%, la utiliza sólo de 1 a 2 veces al mes, mientras que el $28 \%$ de 2 a 4 veces al mes. La Tabla 2 muestra las respuestas de los encuestados. 
Tabla 2. Frecuencia en el uso de las aplicaciones móviles de entrega de comida a domicilio

\begin{tabular}{lcc}
\hline \multicolumn{1}{c}{ Ítem } & Frecuencia & Porcentaje \\
\hline 1-2 veces al mes & 40 & $53.3 \%$ \\
2-4 veces al mes & 21 & $28.0 \%$ \\
$5-6$ veces al mes & 8 & $10.7 \%$ \\
$7-8$ veces al mes & 4 & $5.3 \%$ \\
Más de 9 veces al mes & 2 & $2.7 \%$ \\
\hline
\end{tabular}

Nota: Elaboración propia.

Respecto a la variable Precio/Ahorro, el 25.3\% de los encuestados no percibe que puede ahorrar dinero usando las aplicaciones de entrega de alimentos para comprar comida comparando precios ofrecidos en diferentes comercios, seguido del $24 \%$ que se califica indiferente ante esta situación. Sin embargo, el $33.3 \%$ sostiene que le gusta buscar ofertas baratas en diferentes comercios a través de las aplicaciones de entrega de alimentos.

En lo que concierne a la variable Variedad, poco más de la tercera parte, es decir, el $36 \%$, considera que la aplicación de entrega de comida ofrece una variedad de opciones de restaurantes, mientras que el $32 \%$ una variedad de opciones de alimentos y el $36 \%$ una variedad en cuanto a los precios, lo cual implica que entre la variedad sí representa un factor de mediano peso en el uso de dichas aplicaciones.

La variable Higiene por parte de los repartidores y restaurantes que participan en las aplicaciones de entrega de comida, refleja que, el $66.7 \%$ tiene una opinión neutral y el 33.3\% confía en los estándares en el proceso de preparación, entrega y recepción de los alimentos. Cabe señalar que nadie reflejó no tener confianza alguna en cuanto a la higiene se refiere, lo cual es una buena señal para las apps.

En la Tabla 3 se muestra el cambio que han tenido los consumidores en sus hábitos de compra de comida en el último mes, como resultado del confinamiento por COVID-19.

Entre los mismos, destaca que el $66.7 \%$ de los encuestados respondió haber comprado menos en persona a raíz de las recomendaciones y restricciones que se han suscitado por el COVID-19, lo que ha ocasionado que el $44 \%$ compre en menor medida en tiendas aglomeradas, el 36\% compre más artículos de despensa y el $30.7 \%$ haya aumentado su consumo de comida a domicilio, principalmente.

Entre otros cambios, también se encuentra, aunque en menor medida, que el 17.3\% compre más alimentos envasados. Sólo un 9.3\% afirmó que sus hábitos de compra no han cambiado. 
Tabla 3. Cambio en los hábitos de compra de comida en el último mes como resultado de COVID-19

\begin{tabular}{lcc}
\hline \multicolumn{1}{c}{ Ítem } & Frecuencia & Porcentaje \\
\hline Compro menos en persona & 50 & $\mathbf{6 6 . 7 \%}$ \\
Compro más artículos de despensa & 27 & $\mathbf{3 6 . 0 \%}$ \\
Compro más comida cada vez & 8 & $10.7 \%$ \\
Compro menos en tiendas & 33 & $\mathbf{4 4 . 0 \%}$ \\
aglomeradas & 10 & $13.3 \%$ \\
Compro más alimentos congelados & 9 & $12.0 \%$ \\
Compro más artículos para el hogar & 13 & $17.3 \%$ \\
Compro más alimentos envasados & 3 & $4.0 \%$ \\
Compro menos productos frescos & 8 & $10.7 \%$ \\
Presto más atención al envasado & 23 & $\mathbf{3 0 . 7 \%}$ \\
Mayor consumo de comida a & 3 & $4.0 \%$ \\
domicilio & 6 & $8.0 \%$ \\
Compro menos comida al comprar & 7 & $9.3 \%$ \\
No compro comida & & \\
Los hábitos de compra no han & &
\end{tabular}

Nota: Elaboración propia

Respecto a los hábitos alimenticios, la Tabla 4 refleja el cambio de los consumidores en el último mes como consecuencia de COVID-19.

Tabla 4. Cambio en los hábitos alimenticios en el último mes como resultado de COVID-19

\begin{tabular}{lcc}
\hline \multicolumn{1}{c}{ Ítem } & Frecuencia & Porcentaje \\
\hline Más comidas caseras & 54 & $\mathbf{7 2 . 0} \%$ \\
Menos comida a domicilio & 12 & $16.0 \%$ \\
Más meriendas & 5 & $6.7 \%$ \\
Más comidas prefabricadas & 6 & $8.0 \%$ \\
Más comida a domicilio & 22 & $\mathbf{2 9 . 3 \%}$ \\
Como más de lo habitual & 25 & $\mathbf{3 3 . 3} \%$ \\
Más comida saludable que la típica & 17 & $\mathbf{2 2 . 7} \%$ \\
Menos comida saludable que la & 6 & $8.0 \%$ \\
típica & 6 & $8.0 \%$ \\
Comer menos de lo habitual & 0 & $0.0 \%$ \\
Menos meriendas & 10 & $13.3 \%$ \\
cambiado & &
\end{tabular}

Nota: Elaboración propia.

El 72\% de los encuestados respondió que el cambio en sus hábitos alimenticios se ve reflejado al consumir más comidas caseras, el $33.3 \%$ come más de lo habitual como consecuencia de la pandemia, el 29.3\% tiene más consumo de comida a domicilio y el $22.7 \%$ señala consumir más comida saludable que la típica, posiblemente al permanecer más tiempo en casa. 


\section{Discusión}

\section{Segmentación de la muestra}

Como se muestra en la Tabla 5 , los segmentos de mercado 1 y 3 con respecto a las variables Ahorro de tiempo, Actitud hacia los servicios de entrega de comida, Ahorro de dinero y Variedad se encuentran según la gama de colores y medias en una situación óptima, sin embargo, el segmento número 2 carece de algunos aspectos relacionados a las mismas variables, lo cual hizo necesario concentrarse en este segmento para realizar las respectivas estrategias.

Tabla 5. Análisis del clúster

\begin{tabular}{|c|c|c|c|}
\hline & \multicolumn{3}{|c|}{ Clúster } \\
\hline & Segmento1 & Segmento 2 & Segmento 3 \\
\hline $\begin{array}{l}\text { Creo que usar los servicios de aplicación móvil de entrega } \\
\text { de comida es muy útil en el proceso de compra. }\end{array}$ & 3,55 & 2,36 & 4,64 \\
\hline $\begin{array}{l}\text { Creo que el uso de los servicios de aplicación móvil de } \\
\text { entrega de comida me ayuda a lograr cosas más } \\
\text { rápidamente en el proceso de compra. }\end{array}$ & 3,35 & 2,18 & 4,61 \\
\hline $\begin{array}{l}\text { Creo que puedo ahorrar tiempo al usar los servicios } \\
\text { aplicación móvil de entrega de comida en el proceso de } \\
\text { compra. }\end{array}$ & 3,81 & 2,00 & 4,70 \\
\hline $\begin{array}{l}\text { Para mí es importante que la compra de alimentos se } \\
\text { realice lo más rápido posible utilizando los servicios de } \\
\text { aplicación móvil de entrega de comida. }\end{array}$ & 3,52 & 2,18 & 4,58 \\
\hline $\begin{array}{l}\text { Comprar alimentos a través de los servicios aplicación } \\
\text { móvil de entrega de comida es prudente. }\end{array}$ & 3,32 & 2,45 & 4,39 \\
\hline $\begin{array}{l}\text { Comprar alimentos a través de los servicios de aplicación } \\
\text { móvil de entrega de comida es bueno. }\end{array}$ & 3,29 & 2,36 & 4,42 \\
\hline $\begin{array}{l}\text { Comprar alimentos a través de los servicios de aplicación } \\
\text { móvil de entrega de comida es razonable. }\end{array}$ & 3,10 & 2,36 & 4,33 \\
\hline $\begin{array}{l}\text { Comprar alimentos a través de los servicios de aplicación } \\
\text { móvil de entrega de comida es gratificante. }\end{array}$ & 3,16 & 2,36 & 3,85 \\
\hline $\begin{array}{l}\text { Puedo ahorrar dinero usando aplicaciones de entrega de } \\
\text { alimentos para comprar comida comparando los precios } \\
\text { ofrecidos en diferentes tiendas en línea. }\end{array}$ & 2,97 & 2,00 & 3,36 \\
\hline $\begin{array}{l}\text { Me gusta buscar ofertas baratas en diferentes tiendas en } \\
\text { línea cuando compro comida a través de aplicaciones de } \\
\text { entrega de alimentos. }\end{array}$ & 3,77 & 2,27 & 4,06 \\
\hline $\begin{array}{l}\text { La aplicación de entrega de comida ofrece una variedad de } \\
\text { opciones de restaurantes. }\end{array}$ & 4,06 & 2,45 & 4,42 \\
\hline $\begin{array}{l}\text { La aplicación de entrega de comida ofrece una variedad de } \\
\text { opciones de alimentos. }\end{array}$ & 3,77 & 2,45 & 4,45 \\
\hline $\begin{array}{l}\text { Puedo pedir comida con una amplia gama de precios a } \\
\text { través de la aplicación de entrega de comida. }\end{array}$ & 3,84 & 2,73 & 4,48 \\
\hline
\end{tabular}

Nota: Elaboración propia.

El segmento 2 tiene una baja percepción en cuanto al ahorro de tiempo y dinero a través del uso de aplicaciones móviles de entrega de comida (Delivery), para lo cual, se generaron estrategias para reforzar la percepción referente a estas variables. Dentro de las restricciones, destaca que el consumidor tiene entre 17 y 22 años, mayormente es de sexo femenino, con un nivel de estudios de licenciatura, ingresos entre $\$ 4,501$ y $\$ 10,600$. El número de aplicaciones que usa es una, la aplicación de su preferencia es Ubert Eats y a través de esta la comida es el alimento del día que más pide. El gasto es menor a los $\$ 500$ y su principal razón de uso es por las ofertas, promociones 
y descuentos. Este segmento se llama Ahorradores de tiempo y dinero y las estrategias son las siguientes:

Problema 1: Al usar los servicios de la aplicación móvil de entrega de comida, los consumidores no encuentran cosas más rápido en el proceso de compra.

Estrategia: La aplicación móvil deberá hacer un ajuste en su diseño permitiendo que los usuarios realicen un filtro mucho más específico, una alternativa es generar atajos o accesos directos que faciliten lo anterior. Para los restaurantes, la estrategia está orientada hacia la forma en la que muestran los productos, es decir, deben proporcionar información más precisa y menos tediosa, para que, de esta manera, se agilice el proceso de elección y compra del consumidor.

Problema 2: Al usar los servicios de la aplicación móvil de entrega de comida en el proceso de compra los consumidores no perciben que ahorran tiempo.

Estrategia: Dado el perfil demográfico de los consumidores y su rango de edad, lo que menos quieren es que el proceso de búsqueda y compra se extienda, quieren todo con la mayor brevedad posible. Teniendo en cuenta este aspecto, la estrategia está orientada a generar un beneficio por ser paciente, a través de un descuento y/o enfatizar en que los tiempos de espera son necesarios y que guardan relación con la calidad del servicio. A su vez, la app deberá evaluar si algunas opciones son necesarias y de no ser así, eliminarlas u omitirlas para este tipo de consumidores. Si en dado caso la app muestra publicidad es recomendable que se elimine y de igual forma, pueden priorizar los tiempos de entrega para este tipo de consumidores emitiendo una alerta que sea recibida por los restaurantes.

De manera interna, desde la dirección de app, se deberá evaluar si se está cumpliendo con la promesa en los tiempos de entrega y hacer el respectivo seguimiento, de no ser así, penalizar a los socios restauranteros. La estandarización de sus procesos y el seguimiento a los tiempos de realización y de entrega es imprescindible, puesto que, un servicio rápido aumenta la satisfacción de los consumidores.

Problema 3: Al realizar el proceso de compra de alimentos no se percibe como rápido cuando se utilizan los servicios de aplicación móvil de entrega de comida.

Estrategia: Seguir almacenando los datos de los clientes y sólo dejar como opción de relleno los métodos de pago, de ya tenerlos, la aplicación deberá generar mensajes con frases que aumenten la percepción de rapidez en el proceso de compra tales como: Tu compra a un clic, ¡ compra ya!

Problema 4: Al usar las aplicaciones de entrega de alimentos para comprar comida los consumidores comparan los precios ofrecidos en las diferentes tiendas, pero no perciben que ahorran. 
Estrategia: Habilitar una casilla dentro de la app que vaya contendiendo los ahorros por compra acumulados a la fecha, también recordar dentro de cada compra el ahorro en pesos obtenido y seguir enfatizando en las promociones y descuentos diarios. La estrategia para los restaurantes es idear productos comodines con descuentos.

Por otra parte, aunque no se generaron estrategias para los segmentos 1 y 3 , sí resulta importante conocer las restricciones de estos segmentos. El segmento 1, llamado 1@s cazador@s de ofertas, lo conforman consumidores generalmente de sexo femenino, con edad entre los 28 y 32 años, ingresos entre $\$ 4.501$ y $\$ 10.600$, el número de aplicaciones que usa es una y la aplicación que prefiere es Uber Eats y a través de esta, la comida es el alimento del día que más pide, el gasto es de menos de $\$ 500$ pesos y su principal razón de uso es por las ofertas, promociones y descuentos.

El segmento 3, llamado hogareñ@s, son principalmente de sexo femenino, con edad entre los 28 y 32 años, ingresos entre $\$ 10.600$ y $\$ 17.000$, el número de aplicaciones que usa es dos y las que prefieren son Uber Eats y Rappi, siendo la cena el alimento que más pide, el gasto oscila entre \$501 y $\$ 1,000$ pesos y su principal razón de uso es no salir de casa.

\section{Conclusiones}

El perfil de las personas que usan la aplicación móvil de entrega de comida está definido por personas que utilizan sólo una aplicación, por otro lado, la plataforma que más usan es Uber Eats, el momento de mayor frecuencia en su uso es por la noche para pedir alimentos para cenar, el monto promedio que destinan mensualmente es menos de $\$ 500.00$, la razón principal por la cual utilizan la plataforma Uber Eats es para no salir de casa.

Aunque adquirir alimentos por medio de Uber Eats no se ha convertido en un hábito y de ninguna forma los encuestados se sienten dependientes o adictos al uso de la misma, el uso de la plataforma se ha vuelto algo natural, al mismo tiempo, no consideran de utilidad la aplicación móvil en la entrega de alimentos, sin embargo, la posición es totalmente contraria en el momento de la adquisición de alguna otra cosa, ya que el uso de la aplicación les permita ahorrar tiempo.

Los hábitos de los consumidores se han modificado de una manera drástica en cuanto al consumo de alimentos se refiere, debido a la pandemia causada por COVID-19, no sólo porque entre los hábitos está el comprar menos productos de manera presencial, sino que el consumo de alimentos caseros se ha incrementado considerablemente, puesto que, el $72 \%$ se ha visto en esta necesidad tanto por la situación económica como sanitaria que se vive en el país.

Resulta evidente que el comprar menos en tiendas aglomeradas es una prioridad para la gente hoy en día, puesto que el $44 \%$ afirma la disminución 
de su consumo en este tipo de lugares, lo que representa una oportunidad tanto para restaurantes como para las aplicaciones de entrega de alimentos siempre y cuando ofrezcan promociones y descuentos que el usuario perciba como atractivas, ya que éste es uno de los principales factores referido en la encuesta para el uso y la frecuencia de uso de las aplicaciones de entrega de alimentos. Aunado a lo anterior, el consumidor de estas aplicaciones gusta de buscar ofertas baratas en diferentes tiendas en línea cuando compra comida a través de aplicaciones de entrega de alimentos y es un criterio que estas compañías deben considerarse y hasta cierto grado aceptar.

Compete a las aplicaciones ampliar su oferta en cuanta a variedad de opciones de restaurantes, alimentos y precios se refiere, porque el nivel percibido por los consumidores oscila entre $32 \%$ y $36 \%$, lo cual no refleja un evidente beneficio para ellos. Los restaurantes locales, enfocados a ofrecer productos orgánicos, artesanales, vegetarianos y en general, aquellos dirigidos a ciertos nichos, tendrían una oportunidad de incrementar sus ventas mediante la presencia en las aplicaciones móviles. De esta manera, tanto las aplicaciones como los restaurantes locales y los consumidores se verían beneficiados.

Por último, a través de la investigación se logró realizar la segmentación de la muestra obteniendo que, de tres segmentos, el número 2 tiene una percepción negativa de que a través de estas aplicaciones se puede ahorrar tiempo y dinero, mientras que los segmentos de mercado 1 y 3 , con respecto a las variables analizadas, tienen una percepción más favorable.

\section{References:}

1. AMA (2017). Definitions of Marketing. Chicago: ama.org. Recuperado de https://www.ama.org/the-definition-of-marketingwhat-is-marketing/

2. Accenture (28 de abril de 2020). COVID-19 cambiará para siempre el comportamiento de los consumidores. Obtenido de https://www.accenture.com/cl-es/insights/consumer-goodsservices/coronavirus-consumer-behavior-research

3. Accenture (Abril de 2020). How COVID-19 will permanently change consumer behavior. Obtenido de Fast-changing consumer behaviors influence the future of the CPG industry: https://www.accenture.com/_acnmedia/PDF-123/AccentureCOVID19-Pulse-Survey-Research-PoV.pdf\#zoom $=40$

4. Brizek, M. G., Frash, R. E., McLeod, B. M., \& Patience, M. O. (2020). Independent restaurant operator perspectives in the wake of the COVID-19 pandemic. International Journal of Hospitality Management, 93, 102766. https://doi.org/10.1016/j.ijhm.2020.102766

5. Casas, X. (2020). Delivery en la cuarentena: por las comisiones de las apps en los comercios y casas de comida pierden hasta el $25 \%$ del valor 
de cada venta. Infobae Económico. Recuperado de https://www.infobae.com/economia/2020/04/29/delivery-en-lacuarentena-por-las-comisiones-de-las-apps-los-comercios-y-casas-decomidas-pierden-hasta-25-del-valor-de-cada-venta/

6. Cervantes, V. (2019). ¿Cuáles son las apps de comida preferidas en México? Líder Empresarial. Recuperado de https://www.liderempresarial.com/cuales-son-las-apps-de-comidapreferidas-en-mexico/

7. Delivery Apps: Dando respuesta a las necesidades de un nuevo mercado. Conexión Abastur y Montmart Fashion Service. Recuperado de https://www.conexiones365.com/upload/pdf/conexiones365delivery-apps_webinar.pdf

8. Fenton, M., Dibb, S., \& Furnham, A. (2018). Antecedents and consequences of chronic impulsive buying: Can impulsive buying be understood as dysfunctional self-regulation? Psychology and Marketing, 35(3), 175-188. https://doi.org/10.1002/mar.21078

9. Food Insight (2020). Consumer Survey: COVID-19's Impact on Food Purchasing, Eating Behaviors and Perceptions of Food Safety. Recuperado de https://foodinsight.org/consumer-survey-COVID-19simpact-on-food-purchasing/.

10. Forbes México (2020). Forbes Conecta: Servicio a domicilio, un nicho que será fundamental tras el coronavirus. Ciudad de México: Forber.com.mx. https://www.forbes.com.mx/enfermeras-mexicanasluchan-contra-COVID/.

11. Galeano, S. (2019). Uber Eats: líder entre las apps de comida a domicilio en México. Marketing4ecommerce. Recuperado de https://marketing4ecommerce.mx/uber-eats-lider-entre-las-apps-decomida-a-domicilio-enmexico/\#: : :text=Un\%20dato\%20a\%20notar\%20es,de\%20alrededor $\% 20 \mathrm{de} \% 20700 \% 20$ pesos.

12. Gibler, K., \& Nelson, S. (2003). Consumer Behavior Applications to Real. Obtenido de Journal of Real Estate Practice and Education: https://www.researchgate.net/profile/Karen_Gibler/publication/22901 2046_Consumer_Behavior_Applications_to_Real_Estate_Education/ links/5550a31008ae739bdb91f682/Consumer-Behavior-

Applications-to-Real-Estate-Education.pdf

13. Gomes \& Stedefeldt (2020). COVID-19 pandemic underlines the need to build resilience in commercial restaurants' food safety. Food Research International, 109472.

14. González Vaqué (2020). "FOOD POST COVID-19 [Versión 1/5/2020]"" Reseñas Bibliográficas (BoDiAlCo) Iss. Suplemento 
-Coronavirus" (2020) p. $1-35$ Available at: http://works.bepress.com/luis_gonzalez_vaque/291/

15. Mulato, A. (2019, 21 noviembre). La jugosa apuesta de las reinas del delivery en México. Goula. https://goula.lat/la-jugosa-apuesta-de-lasreinas-del-delivery-en-

mexico/\#:\%7E:text=De\%20acuerdo\%20con\%20estimaciones\%20de, de\%20pesos\%20en\%20el\%20pa\%C3\%ADs.

16. Kotler, F. \& Armstrong, G. (2013). Fundamentos de Marketing. México: Pearson.

17. Kotler, P., \& Armstrong, G. (2007). Marketing versión para Latinoamérica. México: Pearson Prentince Hall.

18. Mombiedro, R. (21 de febrero de 2006). Segmentación: La decisión más crítica del marketing. Obtenido de Segmentación y precios: https://es.scribd.com/document/147260443/Fascaculo-3-Finale

19. Mulato, A. (2019). La jugosa apuesta de las reinas del delivery en México. Goula. Recuperado de https://goula.lat/la-jugosa-apuesta-delas-reinas-del-delivery-en-mexico/

20. Muñoz Osores, M., Fernández Palma, J., Acuña Montes, V., \& Urra Videla, K. (29 de Mayo de 2016). Estudio comparativo de las variables externas que influyen en la decisión de compra de artículos deportivos por parte de jóvenes. Obtenido de Revista Academia \& Negocios Vol. 2 (1) 2016 pp. 23-40: https://papers.ssrn.com/sol3/papers.cfm?abstract_id=2804671

21. Association, N. R. (25 de marzo de 2020). New research details early impact of coronavirus pandemic on restaurant industry. Obtenido de https://restaurant.org/articles/news/study-details-impact-ofcoronavirus-on-restaurants

22. Organización Mundial de la Salud (2020). Brote de enfermedad por el Coronavirus (COVID-19)

Obtenido de https://www.paho.org/es/temas/coronavirus/brote-enfermedad-porcoronavirus-COVID-19

23. Organización Panamericana de la Salud (2020). Coronavirus. Obtenido de https://www.paho.org/es/temas/coronavirus

24. Redacción (2019). Mujeres son de Rappi, hombres de Uber Eats. El Financiero. https://www.elfinanciero.com.mx/empresas/mujeres-son-de-rappihombres-de-uber-eats

25. Reporte de la industria de la comida rápida (2018). Seales and Associates. Recuperado de http://mnamexico.com/wpcontent/uploads/2018/09/Comida-R\%C3\%A1pida.pdf 
26. Reveles, A. (2019, 6 marzo). Estudios Fintonic: Mujeres de 30 años son las que más piden comida a domicilio. Fintonic México. https://blog.fintonic.mx/estudios-fintonic-apps-comida-a-domicilio/

27. Reveles, A. (2020, 4 junio). Estudios Fintonic: Cae $74 \%$ consumo en restaurantes por coronavirus; en apps sube hasta $80 \%$. Fintonic México. https://blog.fintonic.mx/estudios-fintonic-consumo-appscomida-durante-covid-19/

28. Schiffman, L., \& Lazar Kanuk, L. (2005). Comportamiento del consumidor. México: Pearson Prentice Hall.

29. Schiffman, LG., \& Wisenblit, J. (2015). Comportamiento del Consumidor (P. EDUCACIÓN (ed.); 11th ed.).

30. Verma \& Gustafsson (2020). Investigating the Emerging COVID-19 Research Trends in the Field of Business and Management: A Bibliometric Analysis Approach. Journal of Business Research.

31. Wayne, D.H., Deborah, J., \& Macinnis, RP. (2018). Comportamiento del consumidor (CENGAGE L. (ed.); 7ta ed.

32. Winsight (2020). COVID-19: Reopening regulations lead to new formats and menu innovations $(6$ - 23). Recuperado de https://www.technomic.com/technomics-take/coronavirusfoodservice-view. 